\title{
Diversity of Pestalotiopsis-Like Species Causing Gray Blight Disease of Tea Plants (Camellia sinensis) in China, Including two Novel Pestalotiopsis Species, and Analysis of Their Pathogenicity
}

\author{
Yuchun Wang, ${ }_{1}$ Fei Xiong, ${ }^{1,2}$ Qinhua Lu, ${ }^{1}$ Xinyuan Hao, ${ }^{1}$ Mengxia Zheng, ${ }^{1}$ Lu Wang, ${ }^{1}$ Nana Li, ${ }^{1}$ Changqing Ding, \\ Xinchao Wang, ${ }^{1,}$ and Yajun Yang ${ }^{1, \dagger}$ \\ ${ }^{1}$ Tea Research Institute, Chinese Academy of Agricultural Sciences, National Center for Tea Improvement, Key Laboratory of \\ Tea Biology and Resources Utilization, Ministry of Agriculture and Rural Affairs of the People's Republic of China, Hangzhou, \\ China \\ ${ }^{2}$ Tea Research Institute, Nanjing Agricultural University, Nanjing, China
}

\begin{abstract}
Several Pestalotiopsis-like species cause gray blight disease in tea plants, resulting in severe tea production losses. However, systematic and comprehensive research on the diversity, geographical distribution, and pathogenicity of pathogenic species associated with tea plants in China is limited. In this study, 168 Pestalotiopsis-like isolates were obtained from diseased tea plant leaves from 13 primary tea-producing provinces and cities in China. Based on a multilocus (internal transcribed spacer, translation elongation factor $1-\alpha$, and $\beta$-tubulin gene region) phylogenetic analysis coupled with an assessment of conidial characteristics, 20 Neopestalotiopsis unclassified isolates, seven Pestalotiopsis species, including two novel (Pestalotiopsis menhaiensis and Pestalotiopsis sichuanensis), four known (Pestalotiopsis camelliae, Pestalotiopsis

chamaeropis, Pestalotiopsis kenyana, and Pestalotiopsis rhodomyrtus) and one indistinguishable species, and three Pseudopestalotiopsis species, including two known (Pseudopestalotiopsis camelliae-sinensis and Pseudopestalotiopsis chinensis) and one indistinguishable species, were identified. This study is the first to evaluate Pestalotiopsis chamaeropis on tea plants in China. The geographical distribution and pathogenicity tests showed Pseudopestalotiopsis camelliae-sinensis to be the dominant cause of gray blight of tea plants in China. In vitro antifungal assays demonstrated that theobromine not only derepressed mycelial growth of the 29 representative isolates but also increased their growth. Correlation analysis revealed a linear positive relationship between the mycelial growth rate and pathogenicity $(P=0.0148)$.
\end{abstract}

The tea plant [Camellia sinensis (L.) O. Kuntze] is an important commercial crop that is widely cultivated in Asia, Africa, and South America, with China being the largest tea-producing country worldwide. Tea gardens spanning 20 Chinese provinces/cities produced 2,414,802 tons of tea in 2016, according to the Food and Agriculture Organization of the United Nations (2019). Thus, the tea industry has significant economic importance in China. The leaf of the tea plant is a major source of nutrients, such as flavonoids (catechins), purine alkaloids (theobromine and caffeine), and L-theanine, but is frequently affected by diseases that cause losses in tea production (Liu et al. 2017; Wang et al. 2016a; Wei et al. 2018).

Gray blight is one of the most serious diseases in all countries that cultivate tea plants and typically affects plants after the second and

${ }^{\dagger}$ Corresponding authors: X. Wang; wangxinchao@caas.cn, and Y. Yang; yjyang@tricaas.com

Y. Wang and F. Xiong contributed equally to this work.

Funding: This work was supported by the Earmarked Fund for the China Agriculture Research System (grant CARS-19), the Major Project of Agricultural Science and Technology in Breeding of Tea Plant Variety in Zhejiang Province (grant 2016C02053-4), the Chinese Academy of Agricultural Sciences through an Innovation Project for Agricultural Sciences and Technology (grant CAAS-ASTIP-2017-TRICAAS), the China Postdoctoral Science Foundation Funded Project (grants 2017M620970 and 2018T110163), and the Zhejiang Province Postdoctoral Science Foundation Funded Project (grant zj2017153).

*The $\boldsymbol{e}$-Xtra logo stands for "electronic extra" and indicates that one supplementary figure and one supplementary table are published online.

The author(s) declare no conflict of interest.

Accepted for publication 5 May 2019.

(C) 2019 The American Phytopathological Society third crop harvestings from June to August (Yamada et al. 2016). This disease has caused damage to $17 \%$ of the crop produced in southern India (Joshi et al. 2009) and a 10 to $20 \%$ yield loss in Japan (Horikawa 1986). Several reports indicate that gray blight has caused a severe loss of crop yield and quality in some tea-growing regions of China (Chen et al. 2018a; Liu et al. 2017; Maharachchikumbura et al. 2016). Typical symptoms of gray blight include the formation of small brown spots that gradually develop into larger, circular to irregular, brown or black lesions on tea leaves that disrupt plant growth (Chen et al. 2018a).

Pestalotiopsis-like species are the pathogenic cause of gray blight in tea plants worldwide (Joshi et al. 2009; Liu et al. 2017; Yamada et al. 2016). Previously, Pestalotiopsis (Pestalotia) theae was considered the primary species causing gray blight of tea plants according to results obtained using traditional identification methods, such as assessment of host association and various conidial characteristics (Joshi et al. 2009; Maharachchikumbura et al. 2013). With the development and application of current molecular biology techniques, Maharachchikumbura et al. (2014) revised the Pestalotiopsis species and established two novel genera, Neopestalotiopsis and Pseudopestalotiopsis, using multilocus DNA sequence analyses and specific color intensities of the median conidial cell; Pestalotiopsis theae was simultaneously placed into the genus Pseudopestalotiopsis. Subsequently, Liu et al. (2017) reported that identifying Neopestalotiopsis species required care; by analyzing the large number of Neopestalotiopsis strains isolated from tea plants in China, the authors found that the shorter branch lengths and poor support values in phylogenetic gene trees resulted in species confusion. Thereafter, Liu et al. (2019) further revised and improved Sporocadaceae by including the genera Neopestalotiopsis, Pestalotiopsis, and Pseudopestalotiopsis. In 2016 to 2017, 12 novel Pestalotiopsis-like species (Pestalotiopsis and Pseudopestalotiopsis) isolated from tea plants in China were successively reported using the recent classification system (Liu et al. 2017; Maharachchikumbura et al. 2016). Interestingly, an endogenous Pestalotiopsis strain (named Pestalotiopsis fici) isolated from tea plants in China is an important source of bioactive natural 
products (Wang et al. 2015). Because China is a country with rich biodiversity and many tea cultivars are grown in different areas, numerous beneficial Pestalotiopsis-like species that are associated with tea plants may not have been discovered to date. Moreover, gaining an understanding of the geographical distribution of Pestalotiopsis-like species and the dominant species causing gray blight in tea plants in China will be very useful for disease control. Therefore, investigations are urgently needed to reidentify and determine the diversity of Pestalotiopsis-like species associated with tea plants in China.

Theobromine (3,7-dimethylxanthine) and caffeine (1,3,7-trimethylxanthine) are the most important purine alkaloids in tea plants. The caffeine of the tea plant is synthesized from theobromine via methylation steps catalyzed by tea caffeine synthase (Xia et al. 2017). Both theobromine and caffeine have potential toxicities (Ashihara et al. 2008). In vitro experiments have demonstrated that caffeine can significantly inhibit the mycelial growth of Pseudopestalotiopsis theae $(50 \%$ effective concentration of $0.973 \mathrm{mg} / \mathrm{ml}$ ) (Zhang et al. 2010). However, the effect of theobromine on the mycelial growth of Pestalotiopsis-like isolates and whether theobromine is involved in tea plant defenses against Pestalotiopsis-like isolates remains unknown.

To clarify the diversity of Pestalotiopsis-like species affecting $C$. sinensis in China, we collected and isolated these pathogens from diseased leaves of common tea cultivars from 13 major teagrowing regions of China. These pathogenic fungi were identified as 10 Pestalotiopsis-like species from genera Pestalotiopsis and Pseudopestalotiopsis and 20 isolates from genus Neopestalotiopsis. We summarized the Pestalotiopsis-like species associated with tea plants and their geographical distributions in China, performed pathogenicity tests on the 29 representative isolates, and further analyzed the effect of theobromine on the growth of the 29 representative isolates.

\section{Materials and Methods}

Isolation and morphological characterization. Diseased tea leaves were collected from 13 major tea-growing provinces or cities in China (Anhui, Fujian, Guangdong, Guangxi, Guizhou, Henan, Hunan, Hubei, Jiangxi, Sichuan, Yunnan, and Zhejiang provinces and Chongqing city). Fungal isolation was performed as described by Cai et al. (2009) using the single-spore isolation technique. Pure isolates were cultivated on potato dextrose agar (PDA) plates at $25^{\circ} \mathrm{C}$ overnight for 7 days. Morphological characterizations and measurements of the daily growth rate of the colonies were performed as described by Maharachchikumbura et al. (2012). A mycelial disc $(5 \mathrm{~mm})$ was taken from 5-day-old cultures, placed on the middle of a new PDA plate, and incubated at $25^{\circ} \mathrm{C}$ overnight. The daily growth rate was calculated after 5 days of growth and was based on values from three replicates. The morphologies of the fungal mycelia and conidia were observed and photographed via light microscopy (Eclipse 80i; Nikon, Tokyo, Japan). The characteristics of each isolate are based on 30 conidia.

Type specimens of new species from this study were deposited in the Mycological Herbarium, Institute of Microbiology, Chinese Academy of Sciences, Beijing, China (HMAS), and ex-type living cultures were deposited in the China General Microbiological Culture Collection Center. The descriptions of the novel species reported in this study were submitted to the MycoBank database (http:// www.mycobank.org).

DNA extraction and PCR amplification. Total genomic DNA was extracted from isolates using the Ezup Column Fungi Genomic DNA Purification Kit (Sangon Biotech Shanghai Co. Ltd., Shanghai, China). Mycelia were collected from the surfaces of 7-day-old colonies. The internal transcribed spacer (ITS), $\beta$-tubulin gene region (TUB2), and a portion of the translation elongation factor $1-\alpha$ gene (TEF) were amplified using the primer pairs ITS5 (5'-GGAAGT AAAAGTCGTAACAAGG-3') and ITS4 (5'-TCCTCCGCTTATT GATATGC-3') (White et al. 1990), T1 (5'- AACATGCGTGAGA TTGTAAGT- $\left.3^{\prime}\right)$ and Bt-2b (5'-ACCCTCAGTGTAGTGACCC TTGGC-3') (Glass and Donaldson 1995), and EF1-728F (5'-CATC
GAGAAGTTCGAGAAGG-3') and EF-2 (5'-GGA(G/A)GTAC CAGT(G/C)ATCATGTT-3') (Carbone and Kohn 1999; O'Donnell et al. 1998), respectively. Each 50- $\mu$ l PCR mixture contained $25 \mu l$ of Premix Taq (Takara Biomedical Technology Co. Ltd., Beijing, China), $22 \mu$ l of $\mathrm{ddH}_{2} \mathrm{O}, 1 \mu \mathrm{l}$ of each primer, and $1 \mu \mathrm{l}$ of genomic DNA. The thermal cycling program was as follows: 3 min of initial denaturation at $95^{\circ} \mathrm{C}$, followed by 35 cycles of $30 \mathrm{~s}$ of denaturation at $94^{\circ} \mathrm{C}$, $30 \mathrm{~s}$ of primer annealing at $52^{\circ} \mathrm{C}, 1 \mathrm{~min}$ of extension at $72^{\circ} \mathrm{C}$, and a final 10 min of extension at $72^{\circ} \mathrm{C}$. PCR purification and DNA sequencing were performed by Shanghai HuaGene Biotech Co. Ltd. (Shanghai, China). All sequences in this study were deposited in the GenBank database.

Phylogenetic analysis. The sequences used to construct the phylogenetic trees were obtained from GenBank. The reference sequences for the species type (holotype, epitype, and ex-epitype) refer to those of Maharachchikumbura et al. (2014) and Liu et al. (2017, 2019), respectively. The accession numbers are listed in Supplementary Table S1. Sequence alignments of three genes (ITS, TEF, and TUB2) were assembled using MAFFT version 7 (Katoh and Toh 2008) and then checked visually and improved manually with MEGA version 6.0 (Tamura et al. 2011). All gaps were treated as missing data. Maximum likelihood analyses were performed using CIPRES Science Gateway version 3.3 (http:// www.phylo.org/), and RAxML-HPC BlackBox was selected with the default parameters (Liu et al. 2015). The nucleotide substitution models for each data set used to construct the phylogenetic trees were estimated in MrModeltest version 2.3 using the Akaike information criterion (Nylander et al. 2008), and a HKY + I + G model with an inverse gamma distribution rate was selected to construct all phylogenic trees. Bayesian analyses were performed on concatenated alignments using MrBayes version 3.2 (Ronquist and Huelsenbeck 2003) as previously described (Wang et al. 2016a). Analysis of six Markov chain Monte Carlo chains based on the full data set was run for $1 \times 10^{7}$ generations and sampled every 100 generations. The first $25 \%$ of the generations were discarded as burnin. Selection of outgroups was based on the method described by Maharachchikumbura et al. (2014), in which Pseudopestalotiopsis theae MFLUCC 12-0055 was used as the outgroup for the genus Neopestalotiopsis, N. saprophytica MFLUCC 12-0282 was used for the genus Pestalotiopsis, and Pestalotiopsis trachicarpicola OP068 was used for the genus Pseudopestalotiopsis. The trees generated were viewed with FigTree version 1.3.1 (Rambaut and Drummond 2008).

Pathogenicity tests. A total of 29 representative isolates were selected for the pathogenicity tests, including one isolate of Pestalotiopsis camelliae (YN1B5), two isolates of Pestalotiopsis chamaeropis (SC3A13 and SC3A10), five isolates of Pestalotiopsis kenyana (GZ1C2, SC6A5, AH1A1, CQ1A1, and HB2A3), two isolates of Pestalotiopsis menhaiensis (YN3A1 and YN3A2), two isolates of Pestalotiopsis sichuanensis (SC3A21 and SC5A8), two isolates of Pestalotiopsis rhodomyrtus (ZH2A15 and HB2A2), eight isolates of Pseudopestalotiopsis camelliae-sinensis (GX1D3, ZJ1A1, HEN1A2, SC2A8, FJ1A5, GD3A1, HUN2A3, and GX1A6), and seven isolates of Pseudopestalotiopsis chinensis (YN2A2, GX1A4, SC6A3, SC5A6, ZJ2A3, ZJ1A3, and SC6A1). Wound inoculation was performed in vitro as described by Solarte et al. (2018). Healthy, mature tea leaves (third leaf) were collected from 6-year-old C. sinensis 'Longjing43' (LJ43), which is one of the most widely grown cultivars in China, and washed with sterile water; the leaves were then disinfected by immersion in $1 \%$ sodium hypochlorite for $3 \mathrm{~min}$, washed three times with sterilized water, and air dried. Every disinfected leaf was wounded five times on both sides of the main vein using sterilized needles $(0.45 \mathrm{~mm}$ in diameter). Subsequently, a 5-mm disc of PDA medium with mycelia from a 5-day-old culture was placed over every wounded and unwounded leaf for each isolate. The disc was removed 2 days after inoculation. Sterile distilled PDA discs with no mycelia were used as controls. The inoculated leaves were placed in a plastic culture dish and cultured in a growth cabinet at $28^{\circ} \mathrm{C}$ with a light cycle of $12 \mathrm{~h}$ of fluorescent light and $12 \mathrm{~h}$ of darkness for 7 days. Virulence was evaluated as the "lesion diameter" (mean diameter) 
N. vitis MFLUCC 15-1265

Neopestalotiopsis sp. SC3A22

Neopestalotiopsis sp. SC3A1 Neopestalotiopsis sp. SC3A20 Neopestalotiopsis sp. SC3A23

Neopestalotiopsis sp. GX1B1

Neopestalotiopsis sp. HB1B1

N. aotearoa CBS 367.54

$0.98-1$. piceana CBS 254.32

N. samarangensis MFLUCC 12-0233

- Neopestalotiopsis sp. GX1A1 Neopestalotiopsis sp. SC2A3

0.98 - Neopestalotiopsis sp. SC2A4

N . keteleeria MFLUCC 13-0915

Neopestalotiopsis sp. SC5A9

11 N. ellipsospora MFLUCC 12-0284

N. ellipsospora MFLUCC 12-0283

- Neopestalotiopsis sp. ZJ1A2

Neopestalotiopsis sp. SC5A14

1- N. ellipsospora CBS 115113

Neopestalotiopsis sp. SC3A3

Neopestalotiopsis sp. SC6A7

Neopestalotiopsis sp. SC6A16

$\left\{\begin{array}{l}N \text {. foedans CGMCC3.9123 } \\ \text { N. saprophytica CBS } 115452 \\ \mathbf{N} \text {. cubana CBS } 600.96\end{array}\right.$

N. saprophytica MFLUCC 12-0282

N. javaensis CBS 257.31

N. Nesopotamica CBS 336.86

N. rosae CBS 101057

Tree scale: $0.01 \longmapsto$

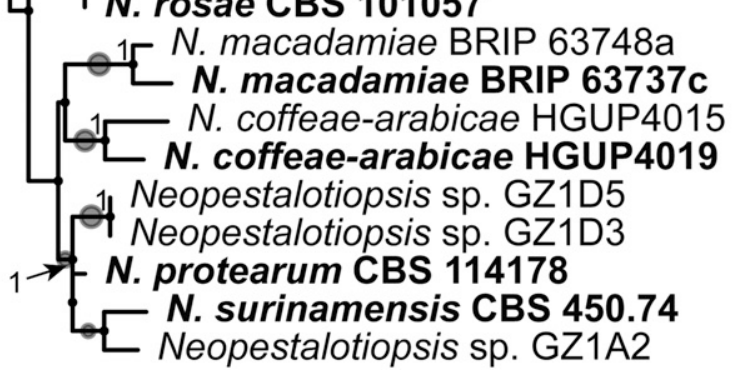

Fig. 1. Maximum-likelihood phylogram generated from the combined sequence alignment (ITS + TEF + TUB) analysis of the Pestalotiopsis isolates. Bootstrap support values $>70 \%$ and Bayesian posterior values $>0.95$ are shown at each node. The circle sizes represent the bootstrap support values. Neopestalotiopsis saprophytica MFLUCC $12-0282$ was used as an outgroup for the genus Pestalotiopsis. Ex-type strains are highlighted in bold. ITS $=$ internal transcribed spacer, TUB2 = $\beta$-tubulin gene region, and TEF = translation elongation factor $1-\alpha$. 
at 3, 5, and 7 days postinoculation. The experiment was repeated twice, and three replicates were included for each treatment (Solarte et al. 2018). Finally, healthy junction tissue was collected from the leaves and cultured on a new PDA plate, and the morphological characteristics of the resulting fungal isolate were checked to confirm Koch's postulates (Cai et al. 2009).

In vitro antifungal bioassay. The antifungal activity of theobromine was assessed using the poison food technique in solid media as described previously (Wang et al. 2016b). Theobromine (Aladdin, Shanghai, China) was added to sterilized PDA $\left(40\right.$ to $\left.50^{\circ} \mathrm{C}\right)$.
The concentrations used for each reagent were $0.0625,0.125$, $0.25,0.5,1$, and $2 \mathrm{mg}$ of theobromine $/ \mathrm{ml}$. Sterile distilled water was used as the control. Mycelial plugs $(5 \mathrm{~mm}$ in diameter) from a 5-day-old culture of each isolate were placed in the centers of the Petri dishes. The inoculated plates were incubated at $25^{\circ} \mathrm{C}$ overnight, and the mycelial growth diameters were measured after 1 day.

Statistical analysis. The mean values of all measurements, including conidium sizes, appressoria, and daily growth rates, were used for statistical analyses. The reported values are expressed as

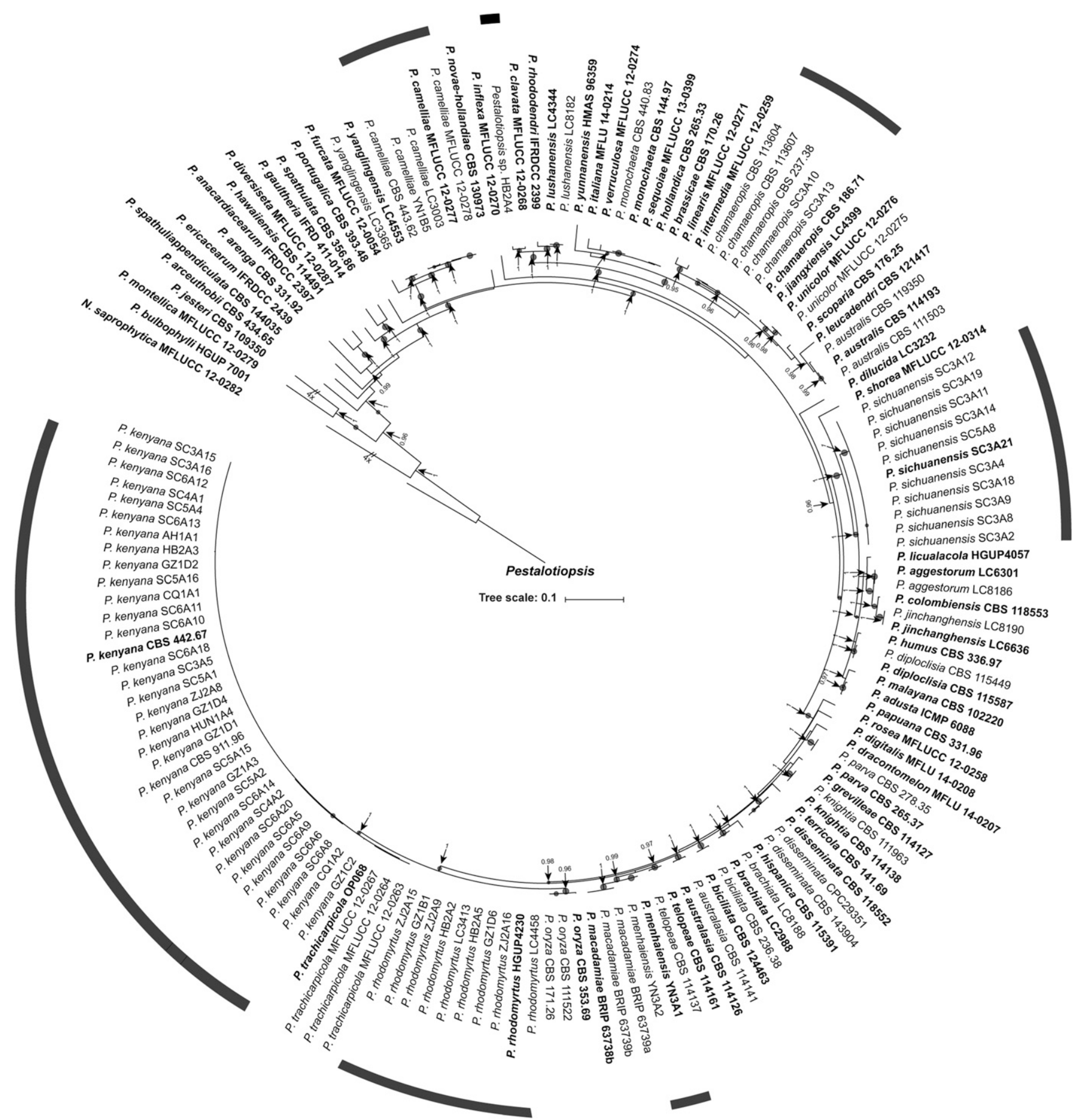

Fig. 2. Maximum likelihood phylogram generated from the combined sequence alignment (ITS + TEF + TUB) analysis of the Pseudopestalotiopsis isolates. Bootstrap support values $>70 \%$ and Bayesian posterior values $>0.95$ are shown at each node. The circle sizes represent the bootstrap support values. Pestalotiopsis trachicarpicola OP068 was used as an outgroup for the genus Pseudopestalotiopsis. Ex-type strains are highlighted in bold. ITS $=$ internal transcribed spacer, TUB2 $=\beta$-tubulin gene region, and TEF $=$ translation elongation factor $1-\alpha$. 
the mean \pm SE. The geographic distribution was produced by ArcGIS 10.5. The data were analyzed with SPSS Statistics version 18 using one-way analysis of variance $(P<0.05)$. Correlation analyses were carried out to show the relationships between mycelial growth and pathogenicity using GraphPad Prism $6(P<0.05)$.

\section{Results}

Phylogenetic analysis. In total, 168 isolates were obtained from the diseased leaves of tea plants from 13 provinces and cities in China. Based on the multigene phylogenetic analysis (ITS, TEF, and TUB2) and the initial morphological characterization, 20, 56, and 92 isolates were identified as members of the genera Neopestalotiopsis (11.9\%), Pestalotiopsis (33.3\%), and Pseudopestalotiopsis (54.8\%), respectively. Among them, Pseudopestalotiopsis camelliae-sinensis was the most common species (56) and accounted for $32.1 \%$ of all isolates (Figs. 1, 2, and 3).

The concatenated data set contained 61 sequences from genus Neopestalotiopsis including the outgroup (Pseudopestalotiopsis theae MFLUCC 12-0055), and 477, 492, and 435 bases had alignment gaps in ITS, TEF, and TUB2, respectively. The 20 isolates obtained from $C$. sinensis in our study clustered with known Neopestalotiopsis species. Among them, Neopestalotiopsis sp. YN1A5 clustered with $N$. asiatica, five Neopestalotiopsis isolates clustered with N. clavispora (FJ1A3, SC3A22, SC3A1, SC3A20, and SC3A23), two isolates clustered with $N$. aotearoa (GX1B1 and HB1B1), three isolates clustered with $N$. samarangensis (GX1A1, SC2A3, and $\mathrm{SC} 2 \mathrm{~A} 4$ ), one isolate clustered with $N$. keteleeria (SC5A9), five isolates clustered with $N$. ellipsospora (ZJ1A2, SC5A14, SC3A3, SC6A7, and SC6A16), two isolates clustered with $N$. protearum (GZ1D3 and GZ1D5), and one isolate clustered with $N$. surinamensis (GZ1A2). However, the topology of the phylogenetic tree was unstable and had low bootstrap support $(<50)$ and Bayesian posterior probability values $(<0.95)$, resulting in these Neopestalotiopsis isolates from tea plants being unclassified with the known species (Fig. 1). Therefore, these Neopestalotiopsis isolates were temporarily unidentified in our study.

The concatenated data set contained 155 sequences from genus Pestalotiopsis including the outgroup ( $N$. saprophytica MFLUCC 12-0282), and 519, 543, and 477 bases had alignment gaps in ITS, TEF, and TUB2, respectively. The 56 Pestalotiopsis isolates obtained from $C$. sinensis in our study distinctly clustered with seven clades with high bootstrap support and posterior probability values ( $\geq 70$ and 0.95 , respectively), including four known species (one isolate of Pestalotiopsis camelliae, two of Pestalotiopsis chamaeropis, 33 of Pestalotiopsis kenyana, and six of Pestalotiopsis rhodomyrtus), two distinct clades (two of Pestalotiopsis menhaiensis and 11 of Pestalotiopsis sichuanensis), and one undefined species (Pestalotiopsis sp. HB2A4) (Fig. 2).

The concatenated data set contained 120 sequences from genus Pseudopestalotiopsis including the outgroup (Pestalotiopsis

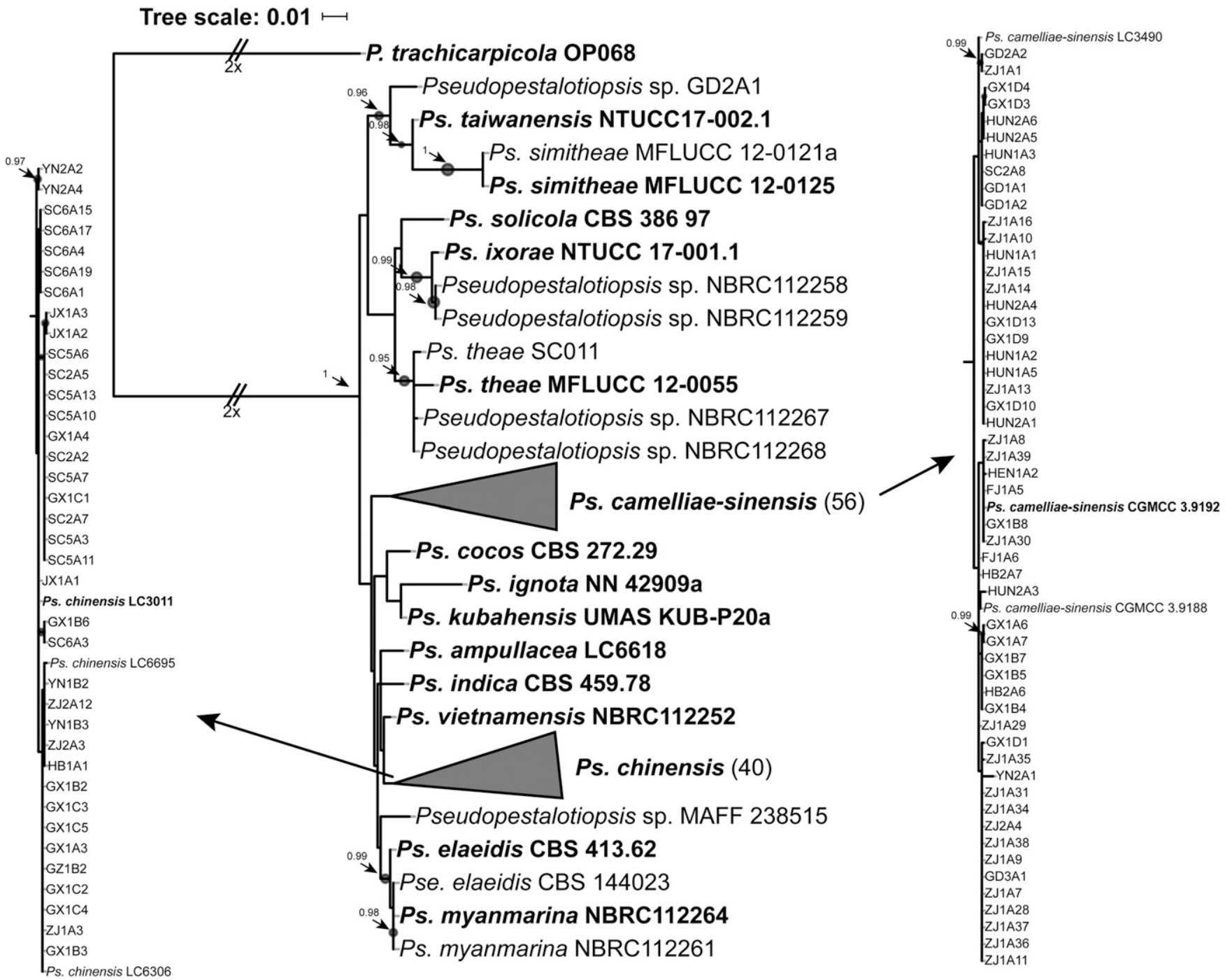

Fig. 3. Maximum-likelihood phylogram generated from the combined sequence alignment (ITS + TEF + TUB) analysis of the Neopestalotiopsis isolates. Bootstrap support values $>70 \%$ and Bayesian posterior values $>0.95$ are shown at each node. The circle sizes represent the bootstrap support values. Pseudopestalotiopsis theae MFLUCC 12-0055 was used as an outgroup for the genus Neopestalotiopsis. Ex-type strains are highlighted in bold. ITS = internal transcribed spacer, TUB2 = $\beta$-tubulin gene region, and TEF = translation elongation factor $1-\alpha$. 
trachicarpicola OP068), and 426, 489, and 376 bases had alignment gaps in ITS, TEF, and TUB2, respectively. The 92 Pseudopestalotiopsis isolates obtained from $C$. sinensis in our study distinctly clustered with high bootstrap support and posterior probability values $(\geq 70$ and 0.95 , respectively) into three clades, including two known species (54 isolates of Pseudopestalotiopsis camelliae-sinensis and 37 of Pseudopestalotiopsis chinensis) and one undefined species (Pseudopestalotiopsis sp. GD2A1) (Fig. 3).

Taxonomy. Species name. Pestalotiopsis chamaeropis. Maharachch., K. D. Hyde \& Crous, Stud. Mycol. 79:158. 2014.
Materials examined. China, Sichuan Province, Hongya County, from diseased leaves of $C$. sinensis 'Mingshan131', 10 September 2014, Y. C. Wang, culture SC3A10; ibid, culture SC3A13 = CGMCC3.18237.

Notes. Pestalotiopsis chamaeropis was first identified on the leaves of Chamaerops humilis. This report is the first of this species on $C$. sinensis. Species name. Pestalotiopsis menhaiensis. Y. C. Wang, X. C. Wang, \& Y. J. Yang sp. nov., MycoBank MB 827687. Figure 4A.

Etymology. Named after the location where it was collected, Menhai City, Yunnan Province.
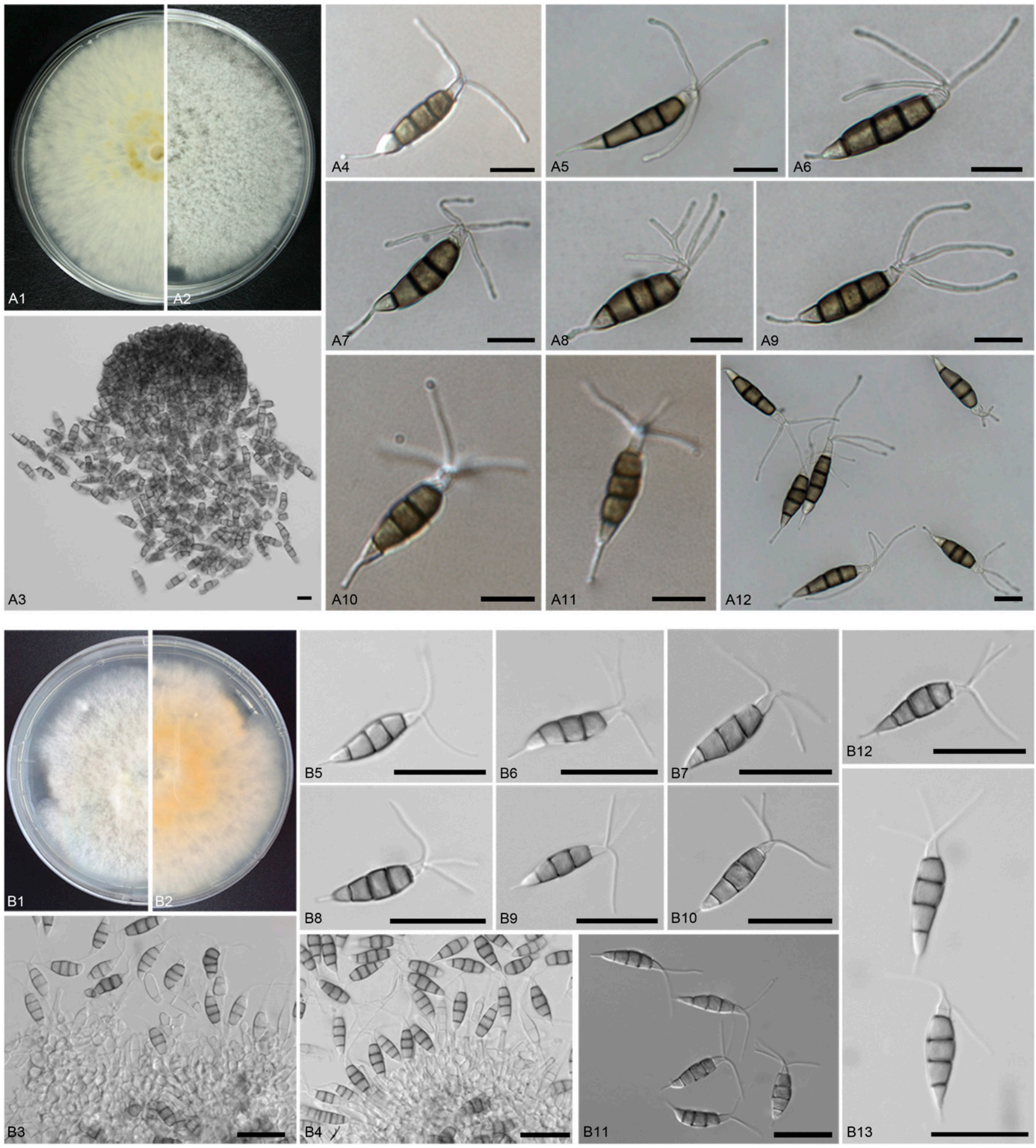

Fig. 4. A, Pestalotiopsis menhaiensis (culture CGMCC3.18250) and B, Pestalotiopsis sichuanensis (culture CGMCC3.18244). A1, A2, B1, and B2, Colonies on potato dextrose agar (PDA) (A1 and B1 from above, A2 and B2 from below). A3, Conidioma sporulating on PDA. B3 and B4, Conidiogenous cells and conidia. A4 to A12 and B5 to B13, Conidia. Scale bars $=10 \mu \mathrm{m}$. 
Known distribution. Yunnan Province in China.

Culture characteristics. Colonies on PDA attain a 70.7-mm diameter after 7 days at $25^{\circ} \mathrm{C}$, with entire edge, whitish, with dense aerial mycelia on the surface with black, solitary conidiomata; reverse whitish in color. Conidiophores were not observed. Conidia ellipsoid or clavate, straight to slightly curved, four-septate, smooth-walled, 21.3 to $28.1 \times 4.7$ to $7.7 \mu \mathrm{m}$, mean $\pm \mathrm{SD}=24.2 \pm 0.5 \times 6.8 \pm$ $0.2 \mu \mathrm{m}$; basal cell obconic to hemispherical, verruculose and thinwalled, 2.9 to $6.2 \mu \mathrm{m}$ long; three median cells doliiform to cylindrical, constricted at the septa, concolorous, brown, septa and periclinal walls darker than the rest of the cell, wall rugose, together 12.9 to $17.1 \mu \mathrm{m}$ long, mean $\pm \mathrm{SD}=15.5 \pm 0.3 \mu \mathrm{m}$ (second cell from the base 4.3 to $5.8 \mu \mathrm{m}$ long; third cell 4.2 to $6.0 \mu \mathrm{m}$ long; fourth cell 4.0 to $5.8 \mu \mathrm{m}$ long); apical cell 3.4 to $5.5 \mu \mathrm{m}$ long, hyaline, obconic or cylindrical to subcylindrical, thin- and smooth-walled; with two to four tubular apical appendages (mostly three), arising from the apical crest, swollen at the tip, flexuous, branched, 10.0 to $15.0 \mu \mathrm{m}$ long; a basal appendage filiform, unbranched, centric, 3.7 to $6.3 \mu \mathrm{m}$ long.

Material examined. China, Yunnan Province, Menhai City, from diseased leaves of $C$. sinensis, 10 September 2014, Y. C. Wang, HMAS 247951 (holotype), ex-holotype living culture YN3A1 = CGMCC3.18250; ibid, culture YN3A2.

Notes. Multilocus (ITS, TEF, and TUB2) phylogenetic tree analysis showed that Pestalotiopsis menhaiensis was a sister clade of the closely related Pestalotiopsis macadamiae, formed a distinct clade with high bootstrap support/posterior probability values (96/0.99), and clustered with Pestalotiopsis telopeae. The morphology of Pestalotiopsis menhaiensis conidia is similar to that of Pestalotiopsis macadamiae, but the conidia of Pestalotiopsis menhaiensis are slimmer $(21.3$ to $28.1 \times 4.7$ to $7.7 \mu \mathrm{m})$ and have shorter apical appendages $(10.0$ to $15.0 \mu \mathrm{m})$ than those of the holotype culture of Pestalotiopsis macadamiae (BRIP 63738b) (conidia: 10.0 to $15.0 \times 10.0$ to $15.0 \mu \mathrm{m}$; apical appendages: 14.0 to 21.0 ). The multiple sequence alignment results showed that the ITS and TEF sequences of Pestalotiopsis menhaiensis (both strains) differ from those of Pestalotiopsis macadamiae (BRIP 63738b) by 1 and $4 \mathrm{bp}$, respectively. In addition, Pestalotiopsis menhaiensis was clearly distinguishable from Pestalotiopsis telopeae by the swelling at the tip of its apical appendages.

Species name. Pestalotiopsis sichuanensis. Y. C. Wang, X. C. Wang, \& Y. J. Yang sp. nov., MycoBank MB 827688. Figure 4B.

Etymology. Named after the province where it was collected, Sichuan.

Known distribution. Sichuan Province in China.
Culture characteristics. Colonies on PDA attain a 73.1-mm diameter after 7 days at $25^{\circ} \mathrm{C}$, edge irregular, whitish, dense aerial mycelium on the surface, with black, gregarious conidiomata; reverse of culture orange. Conidiophores are indistinct, often reduced to conidiogenous cells. Conidiogenous cells integrated, ampulliform or subcylindrical, hyaline, smooth-walled. Conidia fusoid, straight to slightly curved, four-septate, smooth-walled, 8.6 to $12.5 \times 2.6$ to $3.7 \mu \mathrm{m}$, mean $\pm \mathrm{SD}=10.9 \pm 0.9 \times 2.9 \pm 0.3 \mu \mathrm{m}$; basal cell obconic, hyaline, verruculose and thin-walled, 1.7 to $3.0 \mu \mathrm{m}$ long; three median cells doliiform to cylindrical, constricted at the septa, concolorous, brown, septa and periclinal walls darker than the rest of the cell, wall rugose, together 5.3 to $7.6 \mu \mathrm{m}$ long, mean $\pm \mathrm{SD}=6.8 \pm$ $0.6 \mu \mathrm{m}$ (second cell from the base 1.7 to $2.8 \mu \mathrm{m}$ long; third cell 1.6 to $2.6 \mu \mathrm{m}$ long; fourth cell 1.8 to $2.7 \mu \mathrm{m}$ long); apical cell 1.4 to $2.4 \mu \mathrm{m}$ long, hyaline, obconic or subcylindrical, smooth-walled; with two to three tubular apical appendages, arising from an apical crest, unbranched, filiform, flexuous, 2.6 to $9.2 \mu \mathrm{m}$ long; basal appendage single, tubular, unbranched, centric, 1.0 to $8.3 \mu \mathrm{m}$ long.

Material examined. China, Sichuan Province, Hongya County, from diseased leaves of $C$. sinensis 'Mingshan131', 10 September 2014, Y. C. Wang, HMAS 247952 (holotype), ex-holotype living culture SC3A21 = CGMCC3.18244; ibid, culture SC3A11.

Notes. Pestalotiopsis sichuanensis can be easily differentiated from its close relatives in the Pestalotiopsis genus in the TEF and combined phylograms. In the TEF phylogram (maximum likelihood analysis), the Pestalotiopsis sichuanensis isolates are clustered with Pestalotiopsis jesteri CBS 109350, and the TEF gene can clearly separate Pestalotiopsis sichuanensis from other Pestalotiopsis species (Supplementary Fig. S1). In addition, the characteristic morphology of Pestalotiopsis sichuanensis is distinctive within the Pestalotiopsis genus because the conidia are far smaller than those of any other species and it is the smallest known species; the size of Pestalotiopsis knightiae is only 10 to $30 \times 2$ to $10 \mu \mathrm{m}$.

Geographical distribution. To understand the geographical distribution of the Pestalotiopsis-like species associated with $C$. sinensis in China, data from four studies of tea plants in China that used the reliable identification method described by Maharachchikumbura et al. (2014) (Chen et al. 2018a, b; Liu et al. 2017; Maharachchikumbura et al. 2016) and from our study were combined and analyzed. At present, 16 Pestalotiopsis-like species have been identified from tea plants in China. A total of 13 species within the Pestalotiopsis genus were distributed in 11 of 14 provinces/cities, with Pestalotiopsis kenyana as the dominant species (nine provinces/cities). In the Pseudopestalotiopsis genus, three species were distributed in 12 provinces/cities,
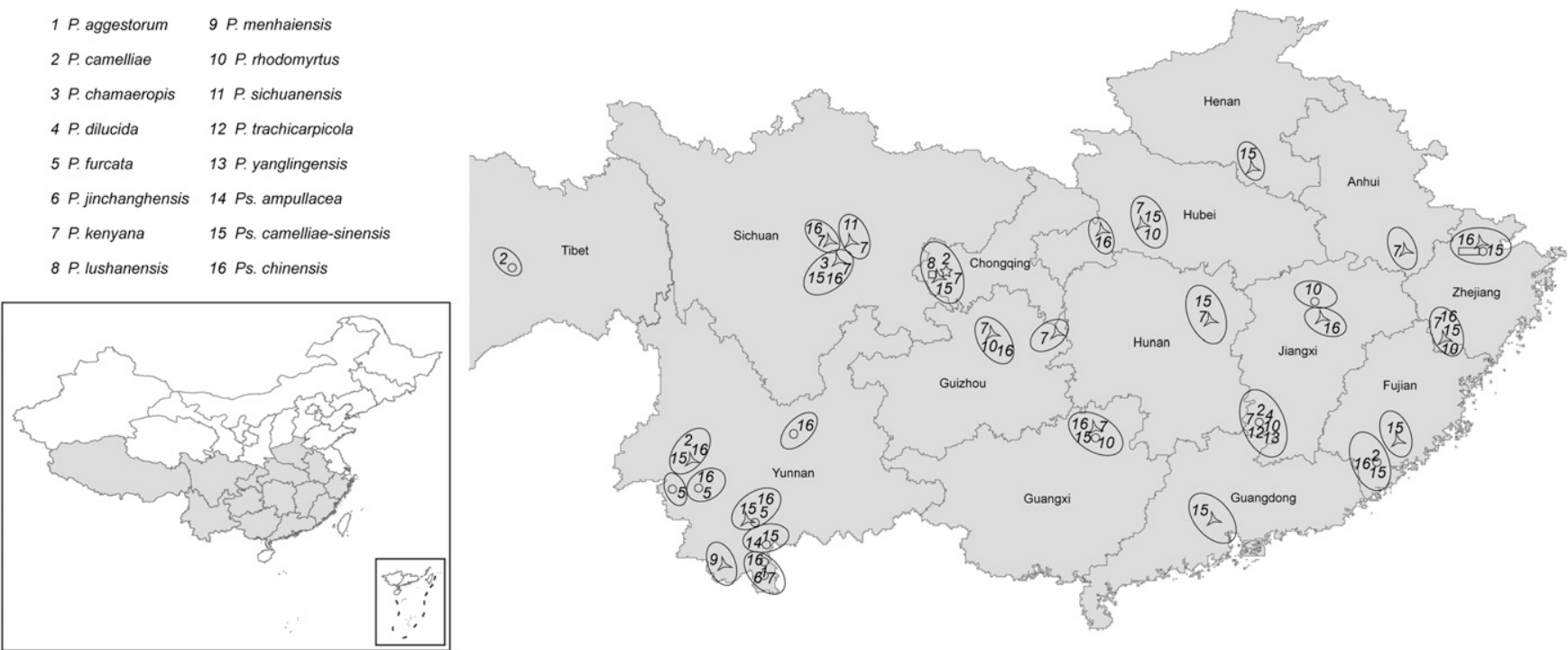

Fig. 5. Geographical distribution of Pestalotiopsis-like species associated with Camellia sinensis in China. These data originate from Chen et al. (2018a) (square), Chen et al (2018b) (pentagrams), Liu et al. (2017) (circles), Maharachchikumbura et al. (2016) (rectangle), and this study (triangles). 
with Pseudopestalotiopsis camelliae-sinensis as the dominant species (10 provinces/cities). These results demonstrate that Pseudopestalotiopsis camelliae-sinensis is the dominant species causing gray blight disease of tea plants in China (Fig. 5).

Pathogenicity assays. The pathogenicity of the 14 Pestalotiopsis and 15 Pseudopestalotiopsis representative isolates (eight classified species) in our study was assessed against LJ43. Upon conducting the pathogenicity tests, symptoms developed within 7 days after the inoculum was detached. All isolates caused notable necrotic gray blight disease lesions around the wounded areas, whereas the unwounded leaves remained unaffected, suggesting that Pestalotiopsis-like isolates might invade tea plants through wounds. All 29 isolates were reisolated from the lesions and cultured on PDA. Their morphological characteristics and ITS gene sequences were the same
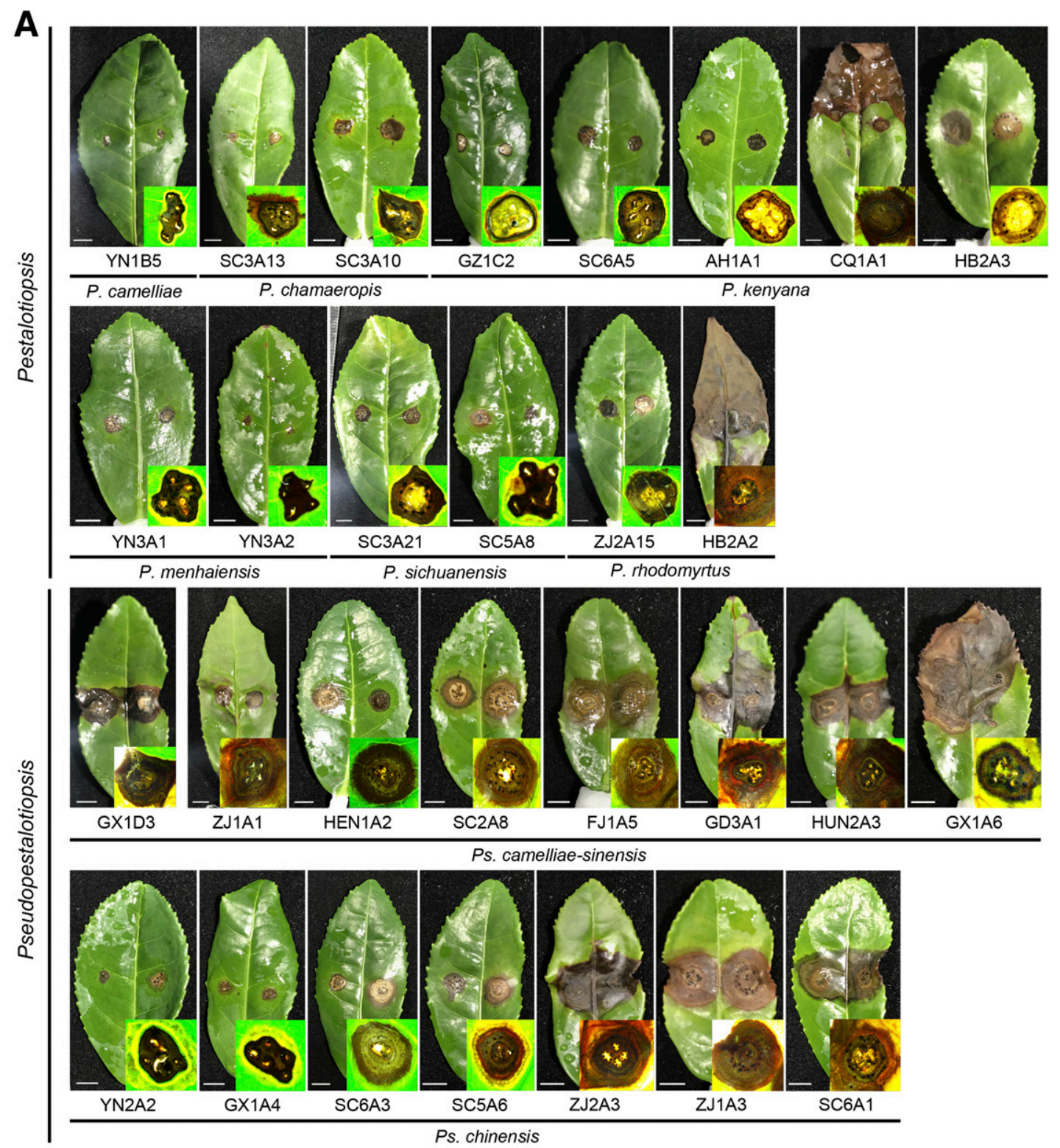

B

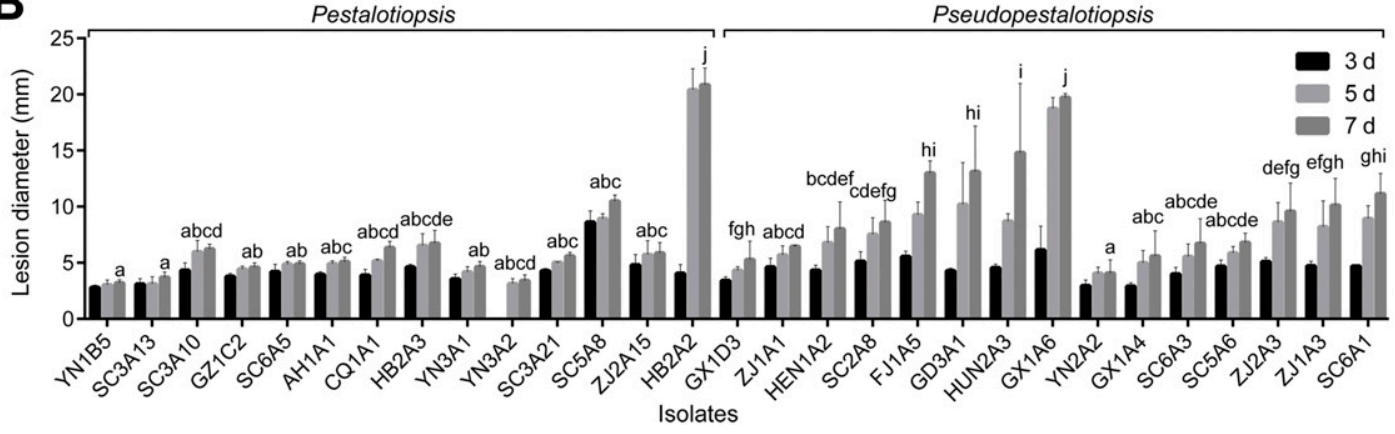

Fig. 6. Pathogenicity of 29 representative Pestalotiopsis-like isolates from tea plants with gray blight in China. A, Induced symptoms on tea leaves after 7 days. B, The virulence of the isolates was evaluated by measuring the diameters of the necrotic lesions on infected tea leaves 7 days after wounding. Values with the same letters in a column do not differ significantly according to Duncan's significant difference test $(P<0.05)$. Scale bars $=5 \mathrm{~mm}$. 
as those of the infecting strains (Fig. 6A). At 7 days after inoculation on wounded leaves, the 14 Pestalotiopsis isolates were pathogenic for the wounded leaves of LJ43, but all isolates except Pestalotiopsis rhodomyrtus (isolate HB2A2) had virulence (lesion diameter: 3.49 to $14.31 \mathrm{~mm}$ ) that was notably weaker than that of the 15 Pseudopestalotiopsis isolates (4.49 to $19.18 \mathrm{~mm}$ ) (Fig. 6B). Meanwhile, the eight representative isolates of Pseudopestalotiopsis camelliae-sinensis ( 5.62 to $19.18 \mathrm{~mm}$ ) had higher virulence than the seven isolates of Pseudopestalotiopsis chinensis (4.49 to $12.63 \mathrm{~mm}$ ) in the Pseudopestalotiopsis genus. These results further confirm that Pseudopestalotiopsis camelliae-sinensis is the causal agent of tea gray blight disease in China.

In vitro effects of theobromine on mycelial growth of Pestalotiopsis-like isolates. The mycelial growth of the 29 representative Pestalotiopsis-like species isolates was examined 1 day after treatment with different theobromine concentrations in vitro (Fig. 7). The results showed that the mycelial growth of these isolates was not only uninhibited after treatment with most of the theobromine concentrations but was even accelerated under the lower concentrations. For example, after treatment with $0.5 \mathrm{mg} / \mathrm{ml}$ of theobromine, the mycelial growth of 21 isolates was accelerated (isolates YN1B5, SC3A13, GZ1C2, AH1A1, CQ1A1, HB2A3, YN3A1, YN3A2, SC3A21, SC5A8, HEN1A2, SC2A8, GD3A1, HUN2A3, GX1A6, YN2A2, GX1A4, SC6A3, SC5A6, ZJ2A3, and SC6A1), that of seven isolates was almost unaffected (SC3A10, SC6A5, ZJ2A15, GX1D3, ZJ1A1, FJ1A5, and ZJ1A3), and that of one isolate was inhibited (HB2A2), suggesting that lower concentrations of theobromine enhanced Pestalotiopsis-like isolate infestation. In addition, we found that the 14 representative Pestalotiopsis species isolates cultured on PDA had smaller colonies (mean colony diameter: $6.81 \mathrm{~mm}$ ) than the 15 Pseudopestalotiopsis species isolates $(11.38 \mathrm{~mm})$ after 1 day of culture.

Correlation analysis between mycelial growth and pathogenicity. The mean values of the 29 representative isolates cultured for 1 day and their lesion diameters at 7 days after inoculation were used to test for a correlation between mycelial growth and pathogenicity. The correlation analysis revealed a significant positive correlation between the colony and lesion numbers (model equation: $Y=0.7727 \times X+1.327$, $r=0.4481, P=0.0148$ ), indicating that the faster-growing isolates had stronger pathogenicity (Fig. 8).

\section{Discussion}

The taxonomy of Pestalotiopsis-like species was previously unclear, primarily because of the diversity of their conidial morphological characteristics, including variable color in the median conidial cells, varying numbers of basal and apical appendages, and the discrepant sizes of the conidial cells (Maharachchikumbura et al. 2011). Currently, accurate DNA data (ITS, TUB, and TEF1) coupled with conidial characteristics are used in scientific studies to identify and classify Pestalotiopsis-like species (Maharachchikumbura et al. 2014). Since the genera Neopestalotiopsis, Pestalotiopsis, and Pseudopestalotiopsis were re-established, at least 92 new species or combinations have been introduced (Liu et al. 2017).
Pseudopestalotiopsis theae was reported to be the dominant species causing gray blight of tea plants in China (Chen et al. 2009), whereas Pestalotiopsis longiseta was identified as the dominant pathogen affecting Japanese tea cultivation (Yamada et al. 2016). In our study, 20 Neopestalotiopsis isolates, seven Pestalotiopsis isolates, and three Pseudopestalotiopsis isolates were identified from a large number of tea cultivars from the primary tea-growing areas in China (Figs. 1, 2, and 3), of which Pseudopestalotiopsis camelliae-sinensis was the dominant pathogen causing gray blight of tea plants (Figs. 5 and 6). However, Pseudopestalotiopsis theae and Pestalotiopsis longiseta were not identified among these species. Liu et al. (2017) also did not identify Pseudopestalotiopsis theae after collecting symptomatic and asymptomatic tissues from Camellia spp. (related to tea plants) from eight provinces in China. Although Pseudopestalotiopsis theae was first collected from the diseased leaves of tea plants in Taiwan, an epitype of this pathogen was reobtained from the leaves of a tea plant in Thailand owing to the lack of an available ex-type culture and lectotype. We speculated that the reason why Pseudopestalotiopsis theae was not observed after the first report was because this species was a pathogen of tea plants that was distributed only in Taiwan or possibly because of an identification error attributable to a previous reliance on either single ITS sequence data or conidial characteristics (Maharachchikumbura et al. 2011). Similarly, several ambiguous species were reported to have been isolated from tea plants in China in the past, such as Pestalotiopsis hangzhouensis, Pestalotiopsis microspora, Pestalotiopsis heterocornis, Pestalotiopsis virgatula, Pestalotiopsis fici, Pestalotiopsis sinensis, Pestalotiopsis versicolor, Pestalotiopsis photiniae, and

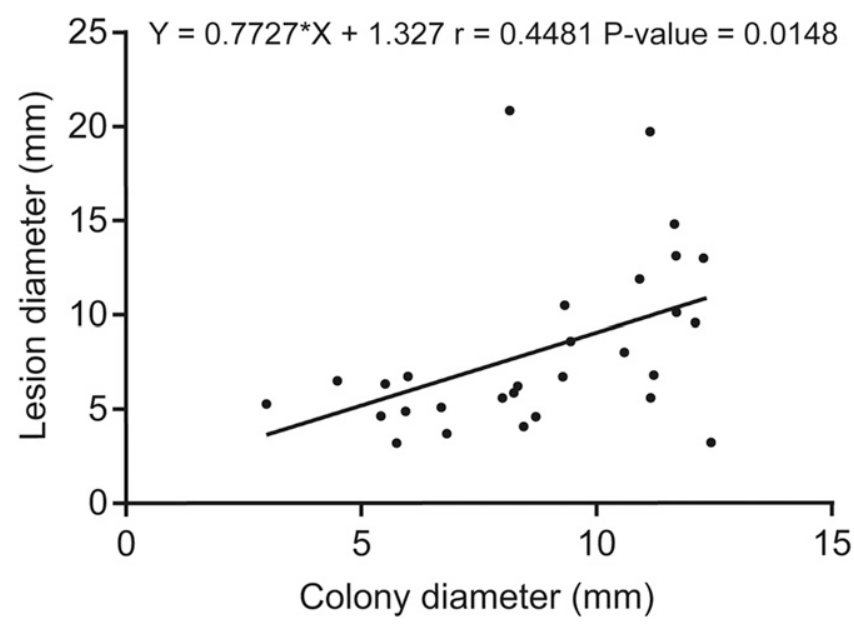

Fig. 8. Correlation analysis between the mycelial growth rates and lesion diameters (model equation: $Y=0.7727 \times X+1.327, r=0.4481, P=0.0148$ ). The mean numbers of colonies of the 29 isolates at 1 day after culture on potato dextrose agar were used as the mycelial growth rates. The mean lesion diameters were determined 7 days after inoculation.

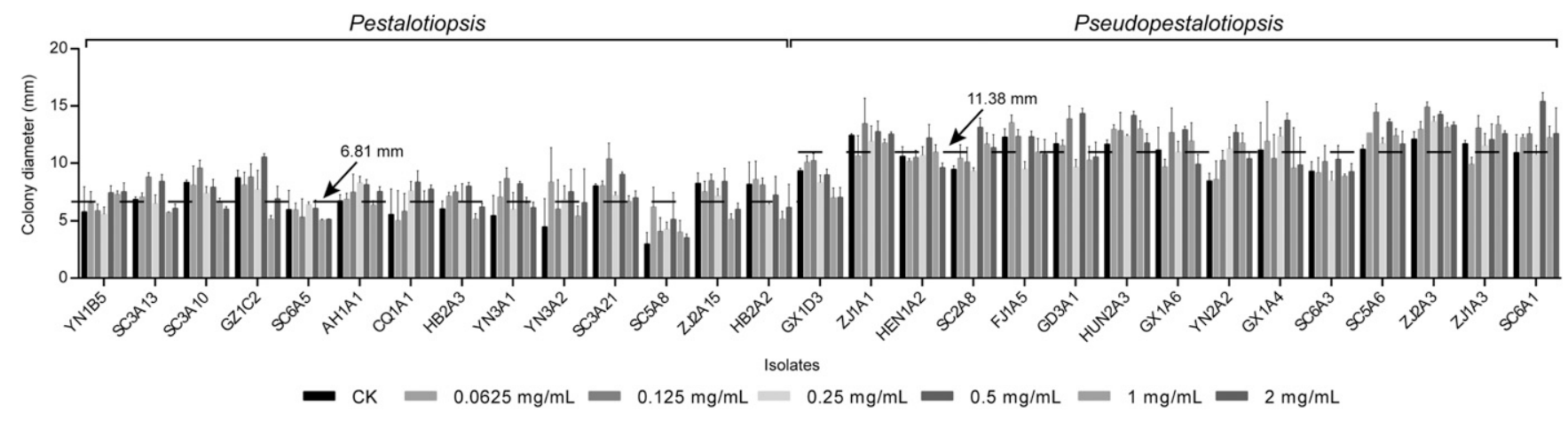

Fig. 7. In vitro effects of theobromine on the mycelial growth of 29 representative Pestalotiopsis-like isolates. $\mathrm{CK}=$ sterile distilled water. 
Pestalotiopsis algeriensis (Wei et al. 2007; Wu 2006). Therefore, further study is necessary to expand the investigated regions and collect Pestalotiopsis-like species from tea plants in China in order to clarify their diversity based on the currently accepted classification system.

The use of molecular data can effectively identify the taxa of Pestalotiopsis-like species (Jayawardena et al. 2016; Maharachchikumbura et al. 2014, 2016; Song et al. 2014). However, Liu et al. (2017) proposed a new perspective, suggesting that the widely used loci to identify Pestalotiopsis-like genera at present do not consistently provide stable and sufficiently resolved tree topologies, especially for the genus Neopestalotiopsis. In our study, 20 isolates were not well distinguished as a result of unstable tree topologies and a lack of samples (Fig. 1). Thus, we also suggest that the loci used to identify Pestalotiopsis-like genera need to be improved in the future.

Pestalotiopsis-like species are either common pathogens or important endophytes (Liu et al. 2007, 2017; Maharachchikumbura et al. 2011; Wei et al. 2007, 2012; Xu et al. 2010), the latter of which produce various special natural products, including antibiotics, anticancer agents, agrichemicals, and other bioactive compounds (Liu et al. 2011; Schulz et al. 2002). Currently, only Pestalotiopsis fici, which was isolated as an endophyte from the branches of tea plants in China, has been thoroughly studied, and this endophyte can produce 88 secondary metabolites that have various bioactivities confirmed by chemical investigations (Liu 2011; Liu et al. 2008). Interestingly, both our study and that of Liu et al. (2017) suggest that Pseudopestalotiopsis camelliae-sinensis is widely distributed among different tea cultivars in China as a pathogen and an endophyte, and the pathogenicity assays demonstrate that eight Pseudopestalotiopsis camelliae-sinensis isolates exhibit strong virulence (lesion diameter: 5.62 to $19.18 \mathrm{~mm}$ ) (Fig. 6B). These results are in accordance with those of Chen et al. (2018a) (5.7 to $10.8 \mathrm{~mm}$ ). Moreover, we found a linear positive relationship between the mycelial growth rate and pathogenicity (Fig. 8). Therefore, further study is needed to determine whether Pseudopestalotiopsis camelliae-sinensis produces beneficial natural products for tea growth and the mechanism underlying its switch between endophytic and pathogenic lifestyles in tea plants (Newton et al. 2010).

Tea plants contain various secondary metabolites, and caffeine is the most important purine alkaloid (Kato et al. 2000). Kim et al. (2014) summarized the role of caffeine in defense against pathogenic microbes and indicated that caffeine had a common toxic effect on a broad range of organisms at concentrations between 0.05 and $0.3 \%$ (wt/vol solution). A low caffeine concentration can significantly inhibit the growth of pathogenic microbes of tea plants, such as Colletotrichum fructicola ( $2 \mathrm{mg} / \mathrm{ml}$ ) (Wang et al. 2016b) and Pseudopestalotiopsis theae (Zhang et al. 2010). However, the antimicrobial effects of theobromine, which is a synthetic precursor of caffeine, are still unknown. In this study, theobromine did not inhibit the growth of 29 Pestalotiopsis-like isolates in the wide concentration range of 0.0625 to $2 \mathrm{mg} / \mathrm{ml}$. Furthermore, low concentrations of theobromine promoted the growth of all 29 isolates (Fig. 7). Therefore, we speculate that tea cultivars that efficiently convert theobromine into caffeine may have stronger disease resistance.

\section{Conclusion}

We investigated the diversity of Pestalotiopsis-like species associated with $C$. sinensis in China and identified 10 Pestalotiopsis and Pseudopestalotiopsis species, including two novel species and one new record species. We summarized the Pestalotiopsis-like species associated with tea plants and their geographical distributions in China. Pseudopestalotiopsis camelliae-sinensis was the dominant species causing gray blight of tea plants in China. In addition, a low concentration of theobromine in tea plants accelerated Pestalotiopsis-like isolate infection, and the hyphal growth rate was associated with pathogenicity.

\section{Acknowledgments}

The authors thank the Tea Experimental Stations of the China Agriculture Research System in Huangshan, Chongqing, Qingyuan, Tongren, Enshi, Xinyang,
Chansha, Wuxi, Yibing, Leshan, Hanzhong, Quanzhou, Guilin, Pu'er, Lishui, and Nanchang for pathogen collection.

\section{Literature Cited}

Ashihara, H., Sano, H., and Crozier, A. 2008. Caffeine and related purine alkaloids: Biosynthesis, catabolism, function and genetic engineering. Phytochemistry 69:841-856.

Cai, L., Hyde, K. D., Taylor, P. W. J., Weir, B. S., Waller, J. M., Abang, M. M., Zhang, J. Z., Yang, Y. L., Phoulivong, S., Liu, Z. Y., Prihastuti, H., Shivas, R. G., McKenzie, E. H. C., and Johnston, P. R. 2009. A polyphasic approach for studying Colletotrichum. Fungal Divers. 39:183-204.

Carbone, I., and Kohn, L. M. 1999. A method for designing primer sets for speciation studies in filamentous ascomycetes. Mycologia 91:553-556.

Chen, Y., Zeng, L., Shu, N., Jiang, M., Wang, H., Huang, Y., and Tong, H. 2018a. Pestalotiopsis-like species causing gray blight disease on Camellia sinensis in China. Plant Dis. 102:98-106.

Chen, Y., Zeng, L., Meng, Q., and Tong, H. 2018b. Occurrence of Pestalotiopsis lushanensis causing grey blight disease on Camellia sinensis in China. Plant Dis. 102:2654.

Chen, Y. S., Liu, W., Ye, N. X., Yang, J. F., Wei, R. F., and Fang, S. M. 2009 The difference of biological characteristics between Pestalotiopsis theae and Pestalotipsis guepini and observation of pathogenicity. J. Tea Sci. 29:449-455.

Food and Agriculture Organization of the United Nations. 2019. FAOSTAT: Crops. http://www.fao.org/faostat/en/\#data/QC.

Glass, N. L., and Donaldson, G. C. 1995. Development of primer sets designed for use with the PCR to amplify conserved genes from filamentous ascomycetes. Appl. Environ. Microbiol. 61:1323-1330.

Horikawa, T. 1986. Yield loss of new tea shoots due to grey blight caused by Pestalotia longiseta Spegazzini. Bull. Shizuoka Tea Exp. Stn. 12:1-8.

Jayawardena, R. S., Liu, M., Maharachchikumbura, S. S. N., Zhang, W., Xing, Q., Hyde, K. D., Nilthong, S., Li, X., and Yan, J. 2016. Neopestalotiopsis vitis sp. nov. causing grapevine leaf spot in China. Phytotaxa 258:63-74.

Joshi, S. D., Sanjay, R., Baby, U. I., and Mandal, A. K. A. 2009. Molecular characterization of Pestalotiopsis spp. associated with tea (Camellia sinensis) in southern India using RAPD and ISSR markers. Indian J. Biotechnol. 8:377-383.

Kato, M., Mizuno, K., Crozier, A., Fujimura, T., and Ashihara, H. 2000. Caffeine synthase gene from tea leaves. Nature 406:956-957.

Katoh, K., and Toh, H. 2008. Recent developments in the MAFFT multiple sequence alignment program. Brief. Bioinform. 9:286-298.

Kim, Y. S., Choi, Y. E., and Sano, H. 2014. Plant vaccination: Stimulation of defense system by caffeine production in planta. Plant Signal. Behav. 5: 489-493.

Liu, A. R., Xu, T., Guo, L. D., Liu, A. R., and Guo, A. 2007. Molecular and morphological description of Pestalotiopsis hainanensis sp. nov., a new endophyte from a tropical region of China. Fungal Divers. 24:23-36.

Liu, F., Bonthond, G., Groenewald, J. Z., Cai, L., and Crous, P. W. 2019. Sporocadaceae, a family of coelomycetous fungi with appendage-bearing conidia. Stud. Mycol. 92:287-415.

Liu, F., Hou, L., Raza, M., and Cai, L. 2017. Pestalotiopsis and allied genera from Camellia, with description of 11 new species from China. Sci. Rep. 7:866.

Liu, F., Weir, B., Damm, U., Crous, P., Wang, Y., Liu, B., Wang, M., Zhang, M. and Cai, L. 2015. Unravelling Colletotrichum species associated with Camellia: Employing ApMat and GS loci to resolve species in the C. gloeosporioides complex. Persoonia 35:63-86.

Liu, L. 2011. Bioactive metabolites from the plant endophyte Pestalotiopsis fici. Mycology 2:37-45.

Liu, L., Bruhn, T., Guo, L., Götz, D. C. G., Brun, R., Stich, A., Che, Y., and Bringmann, G. 2011. Chloropupukeanolides C-E: Cytotoxic pupukeanane chlorides with a spiroketal skeleton from Pestalotiopsis fici. Nat. Prod. Updates 17:2604-2613.

Liu, L., Tian, R., Liu, S., Chen, X., Guo, L., and Che, Y. 2008. Pestaloficiols A-E, bioactive cyclopropane derivatives from the plant endophytic fungus Pestalotiopsis fici. ChemInform 39:606-613.

Maharachchikumbura, S. S., Chukeatirote, E., Guo, L. D., Crous, P. W., Mckenzie, E. H., and Hyde, K. D. 2013. Pestalotiopsis species associated with Camellia sinensis (tea). Mycotaxon 123:47-61.

Maharachchikumbura, S. S. N., Guo, L. D., Cai, L., Chukeatirote, E., Wu, W. P., Sun, X., Crous, P. W., Bhat, D. J., McKenzie, E. H. C., Bahkali, A. H., and Hyde, K. D. 2012. A multi-locus backbone tree for Pestalotiopsis, with a polyphasic characterization of 14 new species. Fungal Divers. 56:95-129.

Maharachchikumbura, S. S. N., Guo, L. D., Chukeatirote, E., Bahkali, A. H., and Hyde, K. D. 2011. Pestalotiopsis-morphology, phylogeny, biochemistry and diversity. Fungal Divers. 50:167-187.

Maharachchikumbura, S. S. N., Guo, L. D., Liu, Z. Y., and Hyde, K. D. 2016 Pseudopestalotiopsis ignota and Ps. camelliae spp. nov. associated with grey blight disease of tea in China. Mycol. Prog. 15:22.

Maharachchikumbura, S. S. N., Hyde, K. D., Groenewald, J. Z., Xu, J., and Crous, P. W. 2014. Pestalotiopsis revisited. Stud. Mycol. 79:121-186.

Newton, A. C., Fitt, B. D., Atkins, S. D., Walters, D. R., and Daniell, T. J. 2010 Pathogenesis, parasitism and mutualism in the trophic space of microbe-plant interactions. Trends Microbiol. 18:365-373. 
Nylander, J. A., Wilgenbusch, J. C., Warren, D. L., and Swofford, D. L. 2008. AWTY (are we there yet?): A system for graphical exploration of MCMC convergence in Bayesian phylogenetics. Bioinformatics 24:581-583.

O'Donnell, K., Kistler, H. C., Cigelnik, E., and Ploetz, R. C. 1998. Multiple evolutionary origins of the fungus causing Panama disease of banana: Concordant evidence from nuclear and mitochondrial gene genealogies. Proc. Natl. Acad. Sci. USA 95:2044-2049.

Rambaut, A., and Drummond, A. 2008. FigTree: Tree figure drawing tool, version 1.2. 2. Institute of Evolutionary Biology, University of Edinburgh, Edinburgh, Scotland.

Ronquist, F., and Huelsenbeck, J. P. 2003. MrBayes 3: Bayesian phylogenetic inference under mixed models. Bioinformatics 19:1572-1574.

Schulz, B., Boyle, C., Draeger, S., Römmert, A. K., and Krohn, K. 2002. Endophytic fungi: A source of novel biologically active secondary metabolites. Mycol. Res. 106:996-1004.

Solarte, F. A., Muñoz, C. G., Maharachchikumbura, S., and Álvarez, E. 2018. Diversity of Neopestalotiopsis and Pestalotiopsis spp., causal agents of guava scab in Colombia. Plant Dis. 102:49-59.

Song, Y., Maharachchikumbura, S. S. N., Jiang, Y. L., Hyde, K. D., and Wang, Y. 2014. Pestalotiopsis keteleeria sp. nov., isolated from Keteleeria pubescens in China. Chiang Mai J. Sci. 41:885-893.

Tamura, K., Peterson, D., Peterson, N., Stecher, G., Nei, M., and Kumar, S. 2011. MEGA5: Molecular evolutionary genetics analysis using maximum likelihood, evolutionary distance, and maximum parsimony methods. Mol. Biol. Evol. 28:2731-2739.

Wang, X., Zhang, X., Liu, L., Xiang, M., Wang, W., Sun, X., Che, Y., Guo, L., Liu, G., and Guo, L. 2015. Genomic and transcriptomic analysis of the endophytic fungus Pestalotiopsis fici reveals its lifestyle and high potential for synthesis of natural products. BMC Genomics 16:28.

Wang, Y. C., Hao, X. Y., Wang, L., Xiao, B., Wang, X. C., and Yang, Y. J. 2016a. Diverse Colletotrichum species cause anthracnose of tea plants (Camellia sinensis (L.) O. Kuntze) in China. Sci. Rep. 6:35287.

Wang, Y. C., Qian, W. J., Li, N. N., Hao, X. Y., Wang, L., Xiao, B., Wang, X. C., and Yang, Y. J. 2016b. Metabolic changes of caffeine in tea plant (Camellia sinensis (L.) O. Kuntze) as defense response to Colletotrichum fructicola. J. Agric. Food Chem. 64:6685-6693.

Wei, C., Yang, H., Wang, S., Zhao, J., Liu, C., Gao, L., Xia, E., Lu, Y., Tai, Y., and She, G. 2018. Draft genome sequence of Camellia sinensis var. sinensis provides insights into the evolution of the tea genome and tea quality. Proc. Natl. Acad. Sci. USA 115:E4151-E4158.

Wei, J. G., Phan, C. K., Wang, L., Xu, T., Luo, J. T., Sun, X., and Guo, L. D. 2012 Pestalotiopsis yunnanensis sp. nov., an endophyte from Podocarpus macrophyllus (Podocarpaceae) based on morphology and ITS sequence data. Mycol. Prog. 12:563-568.

Wei, J. G., Xu, T., Guo, L. D., Liu, A. R., Zhang, Y., Pan, X. H., Xu, J. G., Guo, T. Liu, L. D., and Zhang, A. R. 2007. Endophytic Pestalotiopsis species associated with plants of Podocarpaceae, Theaceae and Taxaceae in southern China. Fungal Divers. 24:55-74.

White, T. J., Bruns, T., Lee, S., and Taylor, J. W. 1990. Amplification and direct sequencing of fungal ribosomal RNA genes for phylogenetics. Pages 315-322 in: PCR Protocols: A Guide to Methods and Applications. M. A. Innis, D. H. Gelfand, J. J. Sninsky, and T. J. White, eds. Academic Press, San Diego, CA.

Wu, X. 2006. Resource, diversity and taxonomy of endophytic Pestalotiopsis on Camellia sinensis in Hangzhou. Master's thesis, Zhejiang University, Hangzhou, China.

Xia, E. H., Zhang, H. B., Sheng, J., Li, K., Zhang, Q. J., Kim, C., Zhang, Y., Liu, Y., Zhu, T., and Li, W. 2017. The tea tree genome provides insights into tea flavor and independent evolution of caffeine biosynthesis. Mol. Plant 10 866-877.

Xu, J., Ebada, S. S., and Proksch, P. 2010. Pestalotiopsis a highly creative genus: Chemistry and bioactivity of secondary metabolites. Fungal Divers. 44:15-31.

Yamada, K., Sonoda, R., and Ishikawa, K. 2016. Population genetic structure of QoI-resistant Pestalotiopsis longiseta isolates causing tea gray blight. Plant Dis. 100:1686-1691.

Zhang, H., Qi, L., and Zhang, Z. 2010. Antifungal activity of caffeine against fungal pathogens of tea plant. J. Nanjing Agric. Univ. 2:63-67. 